\title{
Pressure-engineered optical properties and emergent superconductivity in chalcopyrite semiconductor $\mathrm{ZnSiP}_{2}$
}

\author{
Yifang Yuan ${ }^{1,2}$, Xiangde Zhu', Yonghui Zhou $\mathbb{0}^{1}$, Xuliang Chen ${ }^{1}$, Chao An ${ }^{3}$, Ying Zhou', Ranran Zhang',

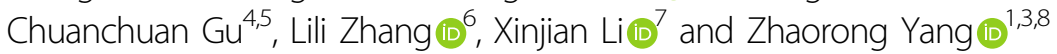

\begin{abstract}
Chalcopyrite II-IV-V $\mathrm{V}_{2}$ semiconductors are promising materials in nonlinear optical, optoelectronic, and photovoltaic applications. In this work, pressure-tailored optical properties as well as pressure-driven emergent superconductivity in chalcopyrite $\mathrm{ZnSiP}_{2}$ are reported via photoluminescence $(\mathrm{PL})$ spectroscopy and electrical transport experiments. During compression, the PL peak energy exhibits a plateau between 1.4 and $8.7 \mathrm{GPa}$, which is accompanied by a piezochromic transition and correlated with the progressive development of cation disorder. Upon further compression across a phase transition from tetragonal to cubic rock-salt structure, superconductivity with a critical temperature $T_{c} \sim 8.2 \mathrm{~K}$ emerges immediately. $T_{c}$ decreases in the range of 24.6-37.1 GPa but inversely increases at higher pressures, thereby exhibiting an unusual V-shaped superconducting phase diagram. These findings present vivid structure-property relationships, which not only offer important clues to optimize the optical and electronic properties, but also provide a new way to use compression to switch between different functionalities.
\end{abstract}

\section{Introduction}

As isoelectronic analogs of III-V zincblende materials, zinc-based chalcopyrites $\mathrm{ZnXPn}_{2}(\mathrm{X}=\mathrm{Si}, \mathrm{Ge}$, and $\mathrm{Sn}$; $\mathrm{Pn}=\mathrm{P}, \mathrm{As}$, and $\mathrm{Sb}$ ) have recently attracted great attention because of their potential technological applications in nonlinear optics, optoelectronics, and photovoltaics ${ }^{1-10}$. For instance, $\mathrm{ZnGeP}_{2}$ can be applied to nonlinear optical devices based on its large nonlinear coefficient, birefringence, and large-area growth availability ${ }^{3} . \mathrm{ZnSnP}_{2}$ is an absorber material for solar cells, whose bandgap can be effectively engineered by tuning the cation disorder ${ }^{6}$. Furthermore, a recent first-principles calculation predicted that $\mathrm{ZnSnP}_{2}$ displays large shift-current

Correspondence: Yonghui Zhou (yhzhou@hmfl.ac.cn) or Xinjian Li (lixj@zzu. edu.cn) or Zhaorong Yang (zryang@issp.ac.cn)

${ }^{1}$ Anhui Province Key Laboratory of Condensed Matter Physics at Extreme Conditions, High Magnetic Field Laboratory, Chinese Academy of Sciences, 230031 Hefei, China

${ }^{2}$ Science Island Branch of Graduate School, University of Science and Technology of China, 230026 Hefei, China

Full list of author information is available at the end of the article conductivity, a bulk photovoltaic phenomenon correlated with the Berry connection between the valence and conduction bands ${ }^{10}$. As for $\mathrm{ZnSiP}_{2}$, it possesses a direct bandgap of $E_{\mathrm{g}} \sim 2.01 \mathrm{eV}^{9}$ and a typically cation-ordered tetragonal structure with intrinsic stability and defect tolerance ${ }^{2}$. Apart from its inexpensive, earth-abundant, and nontoxic elemental constituents, $\mathrm{ZnSiP}_{2}$ has become a promising candidate for transitional tandem solar cells owing to its small lattice mismatch and good refraction index matching with $\mathrm{Si}$, little parasitic below-bandgap absorption, excellent photoresponse, and high opencircuit voltage ${ }^{2,11-13}$. After disorder is introduced into the cation sublattice, $\mathrm{ZnSiP}_{2}$ can be further used as a highperformance anode material for next-generation $\mathrm{Li}$-ion batteries ${ }^{1}$.

As one of the fundamental state parameters, pressure is an effective and clean way to tune the lattice constant, crystal structure, and electronic state, thus varying the fundamental physical properties of materials. Regarding the pressure engineering of photovoltaic and

\section{(c) The Author(s) 2021}

(c) (i) Open Access This article is licensed under a Creative Commons Attribution 4.0 International License, which permits use, sharing, adaptation, distribution and reproduction cc) in any medium or format, as long as you give appropriate credit to the original author(s) and the source, provide a link to the Creative Commons license, and indicate if changes were made. The images or other third party material in this article are included in the article's Creative Commons license, unless indicated otherwise in a credit line to the material. If material is not included in the article's Creative Commons license and your intended use is not permitted by statutory regulation or exceeds the permitted use, you will need to obtain permission directly from the copyright holder. To view a copy of this license, visit http://creativecommons.org/licenses/by/4.0/. 
optoelectronic materials, various exotic phenomena have been revealed recently, including photoluminescence (PL) emission enhancement, prolonged carrier lifetime, bandgap optimization, and superconductivity $^{14-21}$. For the photovoltaic material $\mathrm{ZnSiP}_{2}$, Bhadram et al. ${ }^{22}$ reported that it undergoes a phase transition from tetragonal to cubic rock-salt structure between 27 and $30 \mathrm{GPa}$, in agreement with the ab initio investigations ${ }^{23}$. However, a systematic investigation of the electronic and optical properties of $\mathrm{ZnSiP}_{2}$ under pressure is still lacking to date.

Here, we systematically investigate the pressure effect on the structural, optical, and electronic properties of the chalcopyrite semiconductor $\mathrm{ZnSiP}_{2}$ through various experimental measurements, including synchrotron X-ray diffraction (XRD), Raman spectroscopy, PL spectroscopy, optical microscopy, and electrical transport measurements. We show that pressure-induced modulations in optical and electronic properties correlate well with the structural evolution. For the optical properties, the peak energy of the PL spectrum displays a plateau between 1.4 and $8.7 \mathrm{GPa}$ due to the presence of disorder in the cation sublattice. Moreover, along with the structural phase transition from tetragonal to cubic phase, a V-shaped superconducting behavior is observed.

\section{Materials and methods}

Sample synthesis and characterization at ambient pressure

Single crystals of $\mathrm{ZnSiP}_{2}$ were grown via a flux method $^{24}$. Room-temperature X-ray diffraction (XRD) patterns of single crystals were obtained by using a Rigaku X-ray diffractometer with $\mathrm{Cu} K_{\alpha}$ radiation $(\lambda=1.5406 \AA)$. The atomic proportions of the crystals were characterized by energy dispersive X-ray spectroscopy (EDXS). Absorption spectra were collected by using a UV/Vis/NIR spectrometer (CRAIC 20/30PV).

\section{High-pressure PL spectra, Raman spectra, and X-ray diffraction measurements}

High-pressure PL emission, Raman scattering, and angle-dispersive synchrotron XRD experiments were conducted in symmetric diamond anvil cells (DACs) with rhenium ( $\mathrm{Re})$ as the gasket and silicone oil as the pressure-transmitting medium (PTM). The culet of the diamond was $300 \mu \mathrm{m}$. The PL and Raman spectra were recorded in a Renishaw spectrometer $(\lambda=532 \mathrm{~nm})$ at powers of $0.25 \mathrm{~mW}$ and $2.5 \mathrm{~mW}$, respectively. The dimensions of cleaved single-crystal flakes were $\sim 50 \times$ $50 \times 10 \mu^{3}$. Powder angle-dispersive XRD experiments were carried out at beamline BL15U1 of Shanghai Synchrotron Radiation Facility (SSRF). The wavelength of the monochromatic X-ray beam was $0.6199 \AA$. The Dioptas ${ }^{25}$ and Rietica ${ }^{26}$ programs were employed for image integrations and Le Bail refinements, respectively.

\section{High-pressure electrical transport measurements}

High-pressure transport experiments were performed in a screw-pressure-type DAC made of $\mathrm{CuBe}$ alloy. A pair of anvil culets of $300 \mu \mathrm{m}$ was used. A mixture of epoxy and fine cubic boron nitride ( $c$-BN) powder was compressed firmly to insulate the electrodes from the steel gasket. A single-crystal flake with dimensions of $\sim 120 \times 40 \times 10 \mu \mathrm{m}^{3}$ was loaded together with $\mathrm{NaCl}$ fine powder and ruby powder. A four-probe configuration was utilized to measure the resistance of the flake, where the external magnetic field was perpendicular to the surface of the flake. The ruby fluorescence shift was used to calibrate the pressure at room-temperature in all experiments ${ }^{27}$.

\section{Results and discussion}

The synthesized $\mathrm{ZnSiP}_{2}$ single crystals were characterized via various experimental techniques under ambient conditions. Fig. S1 displays a single-crystal XRD pattern collected from a flake freshly cleaved from a bulk crystal, which shows a (101) orientation of the cleavage plane. The EDXS measurement reveals that the cleaved flake is offstoichiometric with a real composition of $\mathrm{Zn}_{0.92 \pm 0.02} \mathrm{SiP}_{2.07}$ \pm 0.03 . The absorption spectrum shown in Fig. S2a yields a bandgap of $\sim 2.06 \mathrm{eV}$, consistent with that reported in recent literature $^{2}$. The PL spectrum at room-temperature (see Fig. S2b) features a broad emission band peaking at $\sim 919 \mathrm{~nm}$ (equivalent to $1.36 \mathrm{eV}$ ), which can be ascribed to the donor-acceptor pair transitions via defects (vacancies and antisites) $)^{2,6}$. In ref. ${ }^{2}$ Martinez et al. showed that the PL peak energy of $\mathrm{ZnSiP}_{2}$ varies from $1.8 \mathrm{eV}$ to $1.67 \mathrm{eV}$ as the temperature is increased from $5 \mathrm{~K}$ to $100 \mathrm{~K}$, illustrating a temperature effect on the PL peak position. Based on the data of ref. ${ }^{2}$ a value of $\sim 1.4 \mathrm{eV}$ at $300 \mathrm{~K}$ is obtained by extrapolation according to the approximate model for temperature-dependent PL spectra ${ }^{28,29}$. These results consistently confirm the high quality of our samples.

High-pressure synchrotron XRD experiments were conducted on powdered single-crystal $\mathrm{ZnSiP}_{2}$ to explore the structural stability at high pressures. Fig. 1a shows the representative XRD patterns at room-temperature. We note that the peaks at $\sim 15^{\circ}$ and $\sim 17^{\circ}$ arise from the gasket (Re) (see Fig. S3), which persist in the entire pressure region. In addition, all the other peaks progressively shift to larger angles without the appearance of new peaks under compression up to $23.3 \mathrm{GPa}$, indicating the stability of the pristine tetragonal structure. A structural phase transition to the cubic rock-salt type (Fm-3m, No. 225) is detected upon further compression, in agreement with the results of Bhadram et al. ${ }^{22}$ Above $23.3 \mathrm{GPa}$, one can see that the intensity of the peak at $\sim 15^{\circ}$ shows a subtle enhancement, and a broad peak develops at $\sim 22^{\circ}$ in the meantime. The structural transition is complete ca. $36.7 \mathrm{GPa}$, where the high-pressure cubic phase possesses cation disorder, as evidenced by the broad features in the 

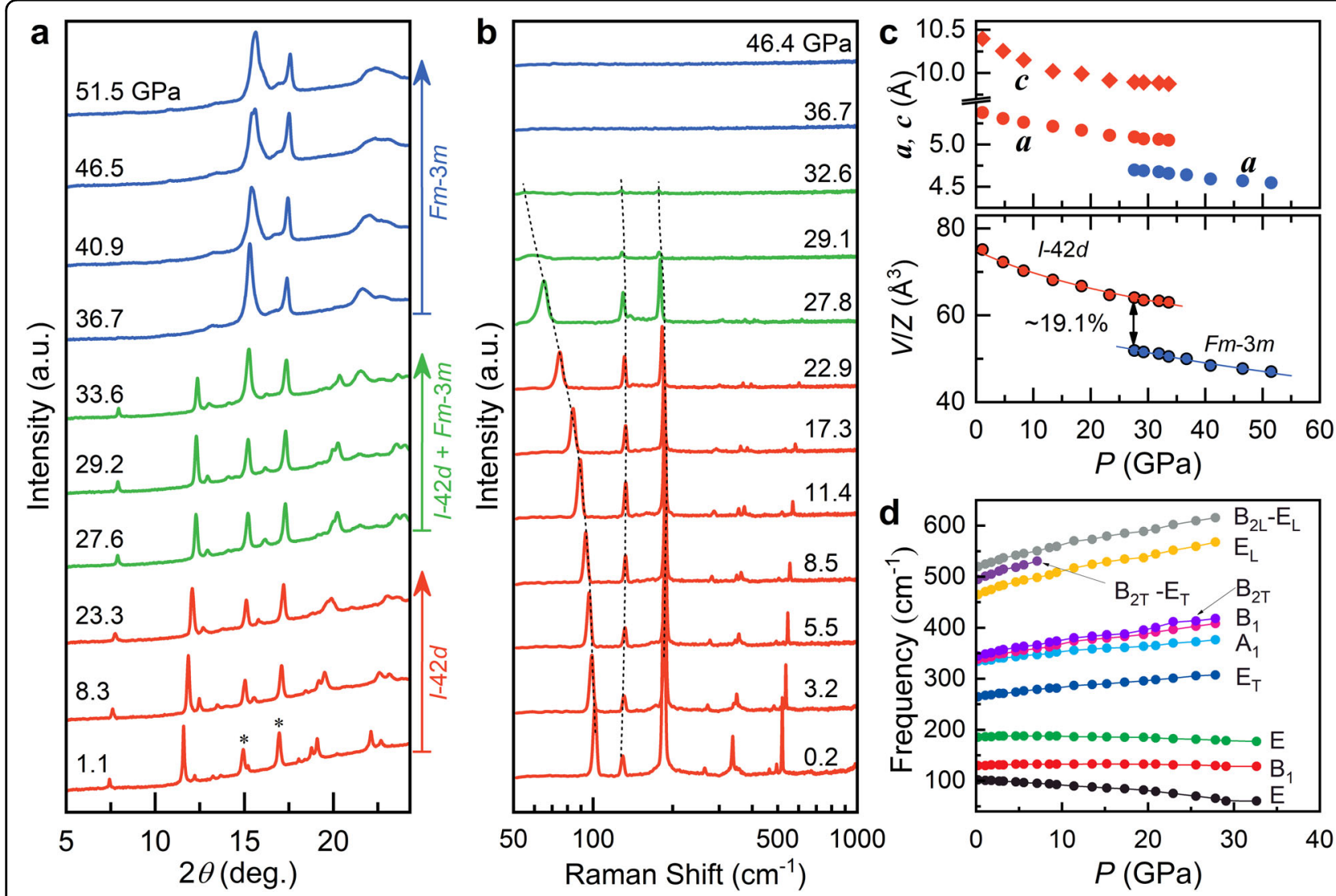

Fig. 1 Structural phase transition of $\mathbf{Z n S i P}_{\mathbf{2}}$ under pressure. a Typical powder synchrotron $X$-ray diffraction patterns at room-temperature $(\lambda=$ $0.6199 \AA)$. The asterisks $(*)$ denote the peaks of the rhenium gasket. b Raman spectra of ZnSiP 2 single crystals at room-temperature $(\lambda=532 \mathrm{~nm})$ with a semilogarithmic scale. c Upper: Pressure-dependent lattice parameters $a$ and $c$ for the pristine tetragonal $(1-42 d, Z=4)$ and high-pressure cubic (Fm$3 m, Z=2)$ phases. Lower: Unit-cell volume as a function of pressure. The solid lines are the fittings according to the third-order Birch-Murnaghan equation of state. $\mathbf{d}$ Pressure-dependent Raman modes of $\mathrm{ZnSiP}_{2}$.

XRD patterns. Although peak broadening in the XRD pattern can be caused by nonhydrostatic compression associated with the PTM, similar broadening behavior was also observed by Bhadram et al. ${ }^{22}$ They used Ar and He as the PTMs, which provide better hydrostatic conditions than the silicone oil used in our case ${ }^{22}$. The extracted lattice parameters $a$ and $c$ are displayed in the upper part of Fig. 1c. The unit-cell volume as a function of pressure can be fitted by the third-order Birch-Murnaghan equation of state ${ }^{30}$; see the solid lines in the lower panel of Fig. 1c. The fittings yield ambient pressure volume $V_{0}=302.0$ (4) $\AA^{3}$, bulk modulus $B_{0}=97.5(6) \mathrm{GPa}$, and first-order derivative of the bulk modulus at zero pressure $B_{0}{ }^{\prime}=6.3$ (0) for the pristine tetragonal phase and 124.9(8) $\AA^{3}, 110.0$ (1) GPa, and 3.4(5) for the high-pressure cubic phase. Note that the errors caused by Le Bail refinements and/or nonhydrostatic conditions are not included for the equation of state fitting. The structural phase transition yields a unit-cell volume contraction $\Delta V / V \sim 19.1 \%$ at 27.6 GPa, similar to the case of a previous report ${ }^{22}$.
Figure $1 \mathrm{~b}$ depicts selective room-temperature Raman spectra of $\mathrm{ZnSiP}_{2}$ at various pressures. At $0.2 \mathrm{GPa}$, the Raman vibrational modes centered at 102.0, 129.9, 185.3, 264.8, 334.4, 338.2, 344.6, 464.8, 494.8, and $519.7 \mathrm{~cm}^{-1}$ can be assigned to $E, B_{1}, E, E_{T}, A_{1}, B_{1}, B_{2 T}, E_{L}, B_{2 T}-E_{T}$, and $B_{2 L}-E_{L}$, respectively ${ }^{31}$. The evolutions of these modes under pressure are qualitatively consistent with those reported in ref. ${ }^{22}$, which were believed to accord with a scenario of a two-stage transition. Based on the model proposed by Bernard and Zunger ${ }^{32}$, it was suggested that $\mathrm{Zn}$ and Si cations substitute each other in the first stage, leading to a partially cation-disordered sublattice in the low-pressure tetragonal phase. The second stage involves the structural transition from the tetragonal phase to the high-pressure cubic phase ${ }^{22}$. The strain energy, set up by the atomic size mismatch between the $\mathrm{Zn}-\mathrm{P}$ and $\mathrm{Si}-\mathrm{P}$ bond lengths, could be the reason to control the nature of the state of order in chalcopyrite $\mathrm{ZnSiP}_{2}{ }^{22,33}$. Owing to the lattice instability caused by progressive development of cation disorder, the modes below $200 \mathrm{~cm}^{-1}$ that 
correspond to acoustic zone-center phonon modes display abnormal evolutions in both frequency and full width at half maximum (FWHM). As shown in Fig. S4, in contrast to the continuous blueshift of optical phonon modes, $B_{1}$ and $E$ demonstrate a crossover from blueshift to redshift ca. 8 and $15 \mathrm{GPa}$, respectively. Meanwhile, the FWHM of each mode shows corresponding changes. Along with the structural transition to the cubic phase upon further compression, these modes show an abrupt reduction in intensity above $27.8 \mathrm{GPa}$ and completely disappear when the structural transition completes at 36.7 GPa. A similar pressure-induced two-stage orderdisorder transition was also claimed in defect chalcopyrite $\mathrm{CdAl}_{2} \mathrm{~S}_{4}{ }^{34}$, nevertheless, direct experimental evidence of the disorder in $\mathrm{ZnSiP}_{2}$ is lacking.

We further investigated the pressure effect on the optical properties of $\mathrm{ZnSiP}_{2}$. Figure 2a displays the PL spectra of $\mathrm{ZnSiP}_{2}$ at different pressures. At $0.7 \mathrm{GPa}$, both the profile and PL peak position are analogous to those at ambient pressure (see Fig. S2b). As the pressure increases up to $8.7 \mathrm{GPa}$, the relative intensity of the PL peak gradually decreases, but the profile remains nearly unchanged. The PL intensity undergoes a strong suppression at 11.7 GPa and becomes almost undetectable at $16.9 \mathrm{GPa}$, as shown in Fig. 2a. In contrast to the common

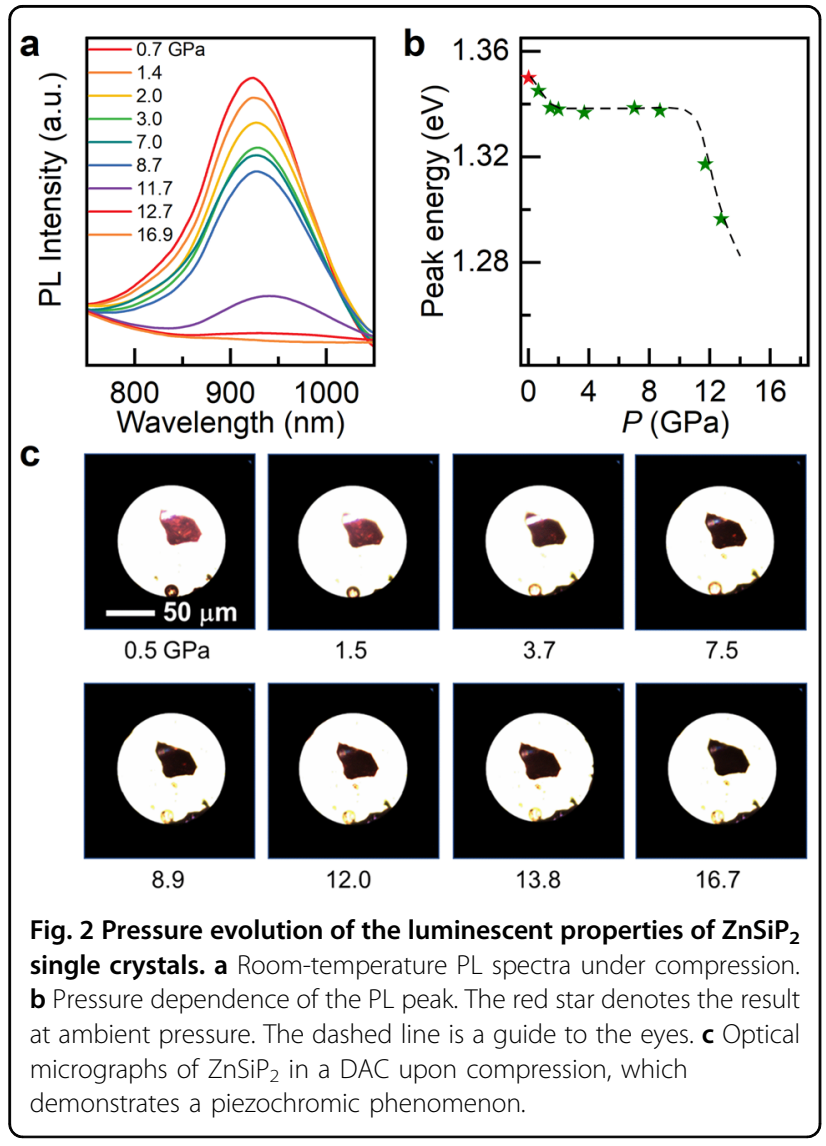

expectation that the peak energy should decrease monotonically with increasing pressure due to the enhancement of orbital overlapping caused by lattice shrinkage, the PL peak energy of $\mathrm{ZnSiP}_{2}$ displays a plateau between 1.4 and 8.7 GPa followed by an abrupt decrease at higher pressures (see Fig. 2b). Note that anomalous evolution of the $\mathrm{PL}$ energy under pressure has been observed in organolead perovskites ${ }^{17,19}$. For example, the blueshift of the PL energy in perovskite (MA) $\mathrm{PbBr}_{3}$ was attributed to pressure-induced amorphization breaking certain bonds ${ }^{17}$. In (MA) $\mathrm{PbI}_{3}$, the abrupt blueshift of the PL energy was ascribed to octahedral tilting, which decreases orbital overlapping ${ }^{19}$. For chalcopyrite $\mathrm{ZnSiP}_{2}$, Martinez et al. ${ }^{2}$ showed that $\mathrm{Si}_{\mathrm{Zn}}{ }^{2+}$ and/or $\mathrm{P}_{\mathrm{Si}}{ }^{1+}$ antisite defects contribute to the donor level, while $\mathrm{Zn}_{\mathrm{Si}}{ }^{2-}$ and/or $\mathrm{Si}_{\mathrm{P}}{ }^{1-}$ antisite defects form the acceptor level. The abnormal plateau could be attributed to the progressive development of cation disorder because donor-acceptor pair transitions are the primary PL mechanism in $\mathrm{ZnSiP}_{2}$. The optical micrographs in Fig. 2c demonstrate a piezochromic transition in compressed $\mathrm{ZnSiP}_{2}$. During the compression process, $\mathrm{ZnSiP}_{2}$ changes from its original transparent red to translucent dark red at $7.5 \mathrm{GPa}$ and eventually turns to opaque black at $16.7 \mathrm{GPa}$.

Because of the insulating nature of $\mathrm{ZnSiP}_{2}$, the resistance at $300 \mathrm{~K}$ is beyond our instrumental limit $\left(10^{7} \Omega\right)$ and could not be detected below 14.0 GPa. Figure $3 \mathrm{a}$ shows the temperature dependence of the resistance $R(T)$ of $\mathrm{ZnSiP}_{2}$ at various pressures up to $55.5 \mathrm{GPa}$. Starting from 14.0 to $21.5 \mathrm{GPa}, \mathrm{ZnSiP}_{2}$ displays semiconducting behavior, as evidenced by the increase in resistance upon cooling. A further increase in pressure leads to a semiconducting-metallic transition at $24.6 \mathrm{GPa}$. Strikingly, the occurrence of metallization is accompanied by a resistance drop below $\sim 8.2 \mathrm{~K}$ (see Fig. $3 \mathrm{~b}$ ). The drop in the $R(T)$ curve becomes increasingly sharper, and zero resistance is finally observed at $37.1 \mathrm{GPa}$, signaling pressure-induced superconductivity in $\mathrm{ZnSiP}_{2}$. Meanwhile, the superconducting critical temperature $T_{\mathrm{c}}$ decreases with increasing applied pressure, reaching a minimum at 37.1 GPa, followed by a continuous increase up to 55.5 GPa, the highest pressure applied in this study. We further measured the $R(T)$ curves under various magnetic fields to determine the upper critical field at 44.4 GPa, as shown in Fig. 3c. By defining $T_{\mathrm{c}}$ as the onset temperature of the superconducting transition, we constructed the temperature-magnetic field phase diagram in the inset of Fig. 3c. According to the Werthamer-Helfand-Hohenberg (WHH) model ${ }^{35}$, the yielded upper critical field $\mu_{0} H_{\mathrm{c}}(0)$ is $\sim 3.0 \mathrm{~T}$. Note that the upper critical field is much lower than the resultant Pauli limiting field of $\mu_{0} H_{\mathrm{P}}(0)=1.84 T_{\mathrm{c}}$, which suggests the absence of Pauli pair breaking. 

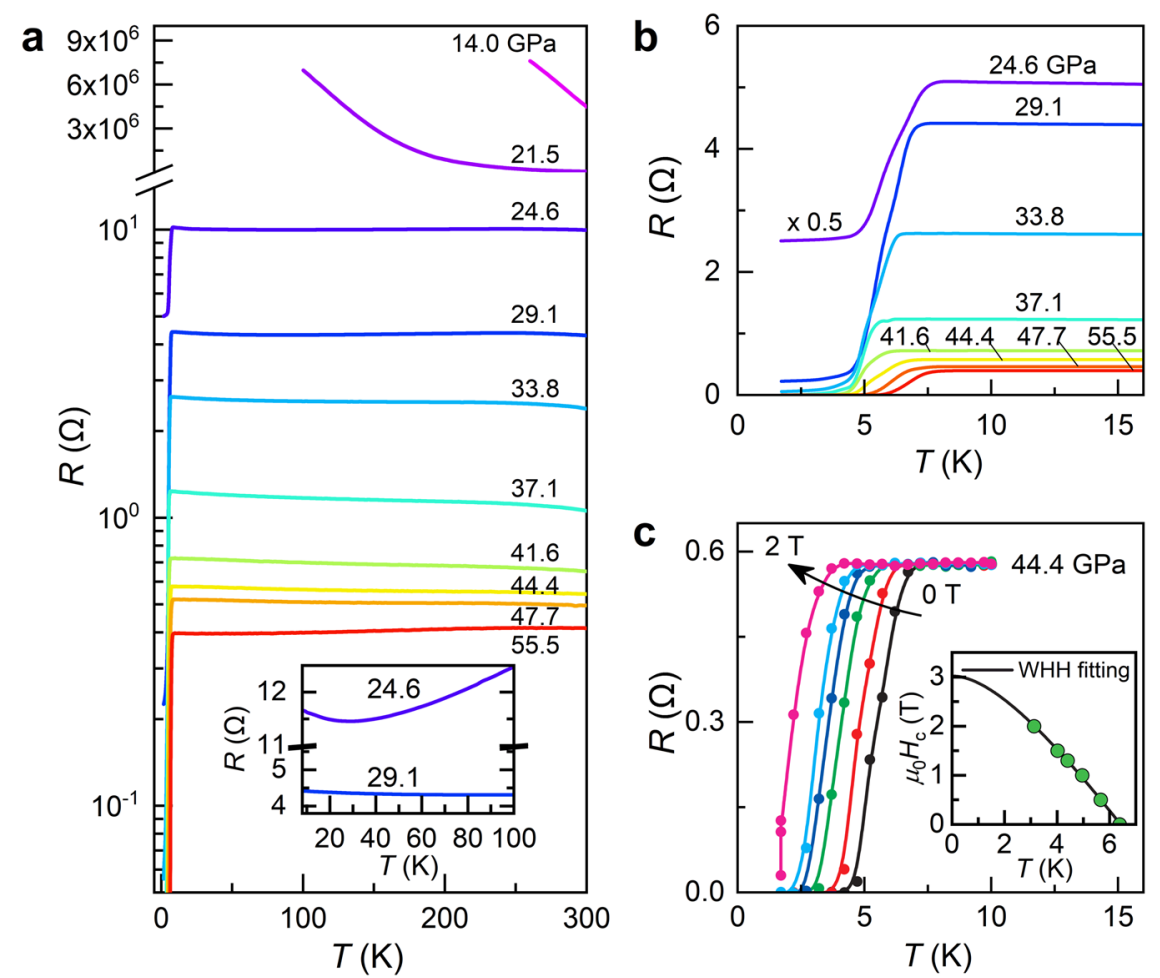

Fig. 3 Experimental evidence of pressure-induced superconductivity in $\mathrm{ZnSiP}_{\mathbf{2}}$. a Selective temperature-resistance curves $R(T)$ of $\mathrm{ZnSiP} \mathrm{P}_{2}$ single crystals under compression. Inset: $R(T)$ curves in the range of 10-100 K. b Low-temperature $R(T)$ curves around the superconducting transition. The curve at $24.6 \mathrm{GPa}$ is multiplied by 0.5 for comparison. c $R(T)$ curves under various magnetic fields at $44.4 \mathrm{GPa}$. Inset: Temperature-dependent upper critical field $\mu_{0} H_{c}$ at $44.4 \mathrm{GPa}$. The solid line represents the fitting based on the WHH model. Here, the $T_{c}$ value was defined as the onset temperature of the superconducting transition.

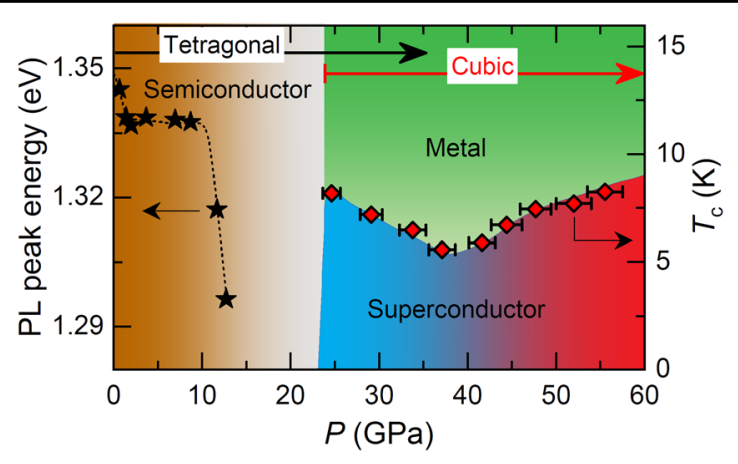

Fig. 4 Pressure evolution of the structural, optical, and electronic properties of $\mathbf{Z n S i P}_{2}$. Pressure evolution of the superconducting transition onset temperature $T_{c}$ and $\mathrm{PL}$ peak energy from $\mathrm{PL}$ analyses. The colored areas are guides to the eyes, indicating the distinct conducting states, i.e., semiconductor, metal, and superconductor. The black and red horizontal arrows indicate the low-pressure tetragonal phase and high-pressure cubic phase, respectively.

To obtain a comprehensive understanding of the evolution of the PL, electrical conductivity, and crystal structure in pressurized $\mathrm{ZnSiP}_{2}$, we constructed a phase diagram, as shown in Fig. 4. It is clear that the appearance of superconductivity can be directly attributed to the structural transition from the tetragonal to cubic phase ca. $23 \mathrm{GPa}$. In the tetragonal phase, $\mathrm{ZnSiP}_{2}$ is situated in the semiconducting regime but possesses the characteristic of cation disorder. As a prelude of the structural transition, the disorder in the cation sublattice gradually develops during compression, leading to the plateau present from 1.4 to $8.7 \mathrm{GPa}$ in the pressure evolution of the PL peak energy. Along with the structural transition above ca. $23 \mathrm{GPa}, \mathrm{ZnSiP}_{2}$ evolves into the superconducting regime. In agreement with the coexistence of tetragonal and cubic phases between 23 and $37 \mathrm{GPa}$, a measurable resistance is still observed at temperatures below the sharp resistance drop. Compared with the XRD results of Bhadram et al., who used $\mathrm{Ar}$ and $\mathrm{He}$ as PTMs, the coexisting pressure range is much larger in our case, which indicates that the coexistence of the two structures can be attributed not only to the incompleteness of the phase transition itself but also to the nonhydrostatic conditions. In the cubic phase, the superconducting phase diagram features an abnormal V-shaped evolution of $T_{\mathrm{c}}$. We note that a similar $\mathrm{V}$-shaped $T_{\mathrm{c}}(P)$ behavior was previously reported in some superconducting compounds, such as $\mathrm{AFe}_{2} \mathrm{As}_{2}$ 
$(\mathrm{A}=\mathrm{K}, \mathrm{Rb}, \mathrm{Cs})^{36,37}, \mathrm{PbTaSe}_{2}{ }^{38}$, and $\mathrm{TlInTe}_{2}{ }^{39}$, while the underlying mechanism is still under debate. On the one hand, the origin of V-shaped $T_{\mathrm{c}}$ behavior has been discussed in terms of a change in the superconducting pairing symmetry or a Lifshitz transition across the critical pressure ${ }^{36,38}$. On the other hand, Yesudhas et al. ${ }^{39}$ observed unusual giant phonon softening ( $\mathrm{A}_{\mathrm{g}}$ mode) concomitant with the $\mathrm{V}$-shaped $T_{\mathrm{c}}(P)$ behavior in $\mathrm{TlInTe}_{2}$. In our case, one can see that the structural phase transition from the tetragonal to cubic phase is almost complete around the valley pressure of $36.7 \mathrm{GPa}$, which implies that the $\mathrm{V}$-shaped behavior might be associated with abnormal evolution of the electron-phonon coupling due to the incompleteness of the phase transition in that pressure regime and/or to the nonhydrostatic conditions.

\section{Conclusions}

In summary, by systematically investigating the pressure effect on the optical and electronic properties, we demonstrate the pressure-induced abnormal evolution of the PL spectrum as well as superconductivity in the chalcopyrite semiconductor $\mathrm{ZnSiP}_{2}$. The abnormal evolution of the PL peak energy is accompanied by a piezochromic transition and is attributed to the gradual development of disorder in the cation sublattice. The superconductivity that shows a $\mathrm{V}$-shaped $T_{\mathrm{c}}(P)$ phase diagram can be directly correlated with the structural phase transition from the tetragonal to cubic phase ca. $23 \mathrm{GPa}$. Based on the fact that a material with optoelectronic and photovoltaic applications is transformed into a superconductor, these findings provide crucial insight into the structure-property relationships in chalcopyrite semiconductors.

\section{Acknowledgements}

This work was supported by the National Key Research and Development Program of China (Grant Nos. 2018YFA0305700 and 2016YFA0401804), the National Natural Science Foundation of China (Grant Nos. U1632275, U1932152, 11874362, 11704387, 11804344, 11804341, 11974016, U19A2093, and U1832209), the Natural Science Foundation of Anhui Province (Grant Nos. 1808085MA06, 2008085QA40, and 1908085QA18), the Users with Excellence Project of Hefei Center CAS (Grant No. 2018HSC-UE012), the Collaborative Innovation Program of Hefei Science Center CAS (Grant No. 2020HSC-CIP014), and the Major Program of Development Foundation of Hefei Center for Physical Science and Technology (Grant No. 2018ZYFX002). Yonghui Zhou was supported by the Youth Innovation Promotion Association CAS (Grant No. 2020443). A portion of this work was supported by the High Magnetic Field Laboratory of Anhui Province. The highpressure synchrotron $X$-ray diffraction experiments were performed at beamline BL15U1, Shanghai Synchrotron Radiation Facility.

\footnotetext{
Author details

${ }^{1}$ Anhui Province Key Laboratory of Condensed Matter Physics at Extreme Conditions, High Magnetic Field Laboratory, Chinese Academy of Sciences, 230031 Hefei, China. ${ }^{2}$ Science Island Branch of Graduate School, University of Science and Technology of China, 230026 Hefei, China. Institutes of Physical Science and Information Technology, Anhui University, 230601 Hefei, China. ${ }^{4}$ Department of Materials Science and Engineering, Southern University of Science and Technology, 518055 Shenzhen, China. ${ }^{5}$ Hefei National Laboratory for Physical Sciences at Microscale, University of Science and Technology of China, 230026 Hefei, China. ${ }^{6}$ Shanghai Synchrotron Radiation Facility, Shanghai Advanced Research Institute, Chinese Academy of Sciences, 201204 Shanghai, China. ${ }^{7}$ Department of Physics and Laboratory of Material Physics, Zhengzhou
}

University, 450052 Zhengzhou, China. ${ }^{8}$ Collaborative Innovation Center of Advanced Microstructures, Nanjing University, 210093 Nanjing, China

Conflict of interest

The authors declare that they have no conflict of interest.

\section{Publisher's note}

Springer Nature remains neutral with regard to jurisdictional claims in published maps and institutional affiliations.

Supplementary information The online version contains supplementary material available at https://doi.org/10.1038/s41427-021-00285-0.

Received: 13 August 2020 Revised: 25 November 2020 Accepted: 9 December 2020.

Published online: 12 February 2021

\section{References}

1. Li, W. et al. A new family of cation-disordered Zn(Cu)-Si-P compounds as high-performance anodes for next-generation Li-ion batteries. Energy Environ. Sci. 12, 2286-2297 (2019).

2. Martinez, A. D. et al. Solar energy conversion properties and defect physics of ZnSiP. Energy Environ. Sci. 9, 1031-1041 (2016).

3. Medvedkin, G. A. \& Voevodin, V. G. Magnetic and optical phenomena in nonlinear optical crystals $\mathrm{ZnGeP}_{2}$ and $\mathrm{CdGeP}_{2}$. J. Opt. Soc. Am. B 22, 1884 (2005).

4. He, G., Rozahun, I., Li, Z., Zhang, J. \& Lee, M.-H. Size effect and identified superior functional units enhancing second harmonic generation responses on the IIIIV-V $V_{2}$ type nonlinear optical crystals. Chem. Phys. 518, 101-106 (2019).

5. Martinez, A. D. et al. Development of $\mathrm{ZnSiP}_{2}$ for Si-based tandem solar cells. IEEE J. Photovolt. 5, 17-21 (2015).

6. Scanlon, D. O. \& Walsh, A. Bandgap engineering of $\mathrm{ZnSnP}_{2}$ for high-efficiency solar cells. Appl. Phys. Lett. 100, 251911 (2012).

7. Sreeparvathy, P. C., Kanchana, V. \& Vaitheeswaran, G. Thermoelectric properties of zinc based pnictide semiconductors. J. Appl. Phys. 119, 085701 (2016).

8. Li, R. et al. High-throughput screening for advanced thermoelectric materials: diamond-like $\mathrm{ABX}_{2}$ compounds. ACS Appl. Mater. Interfaces 11, 24859-24866 (2019).

9. Shay, J. L., Tell, B., Buehler, E. \& Wernick, J. H. Band structure of ZnGeP2 and $\mathrm{ZnSiP}_{2}$ - ternary compounds with pseudodirect energy gaps. Phys. Rev. Lett. 30, 983-986 (1973)

10. Sadhukhan, B., Zhang, Y., Ray, R. \& van den Brink, J. First-principles calculation of shift current in chalcopyrite semiconductor $\mathrm{ZnSnP}_{2}$. Phys. Rev. Mater. $\mathbf{4}$, 064602 (2020).

11. Martinez, A. D., Fioretti, A. N., Toberer, E. S. \& Tamboli, A. C. Synthesis, structure, and optoelectronic properties of II-IV-V $V_{2}$ materials. J. Mater. Chem. A 5, 11418-11435 (2017)

12. Martinez, A. D. et al. Growth of amorphous and epitaxial $\mathrm{ZnSiP}_{2}-\mathrm{Si}$ alloys on Si. J. Mater. Chem. C. 6, 2696-2703 (2018).

13. Liu, H. et al. The pressure-effects on phase transitions, band structures, electronic and elastic properties of ternary compounds $\mathrm{ZnXP}_{2}(X=\mathrm{Si}, \mathrm{Ge}, \mathrm{Sn})$ by first principle calculations. Mater. Res. Express 5, 126303 (2018).

14. Zhang, L. et al. Pressure-induced emission enhancement, band-gap narrowing, and metallization of halide perovskite $\mathrm{CS}_{3} \mathrm{Bi}_{2} 1$. Angew. Chem. Int. Ed. 57, 11213-11217 (2018).

15. Yuan, $Y$. et al. Large band gap narrowing and prolonged carrier lifetime of $\left(\mathrm{C}_{4} \mathrm{H}_{9} \mathrm{NH}_{3}\right)_{2} \mathrm{Pbl}_{4}$ under high pressure. Adv. Sci. 6, 1900240 (2019).

16. Liang, Y. et al. New metallic ordered phase of perovskite $\mathrm{CsPbl}_{3}$ under pressure. Adv. Sci. 6, 1900399 (2019).

17. Wang, Y. et al. Pressure-induced phase transformation, reversible amorphization, and anomalous visible light response in organolead bromide perovskite. J. Am. Chem. Soc. 137, 11144-11149 (2015).

18. Liu, G. et al. Pressure-induced bandgap optimization in lead-based perovskites with prolonged carrier lifetime and ambient retainability. Adv. Funct. Mater. 27, 1604208 (2017).

19. Jaffe, A. et al. High-pressure single-crystal structures of 3D lead-halide hybrid perovskites and pressure effects on their electronic and optical properties. ACS Cent. Sci. 2, 201-209 (2016). 
20. Chi, Z. et al. Superconductivity in pristine $2 \mathrm{H}_{\mathrm{a}}-\mathrm{MoS}_{2}$ at ultrahigh pressure. Phys. Rev. Lett. 120, 037002 (2018)

21. Zhou, Y. et al. Pressure-induced metallization and robust superconductivity in pristine 1T-SnSe2. Adv. Electron. Mater. 4, 1800155 (2018).

22. Bhadram, V. S. et al. Pressure-induced structural transition in chalcopyrite ZnSiP. Appl. Phys. Lett. 110, 182106 (2017).

23. Arab, F., Sahraoui, F. A., Haddadi, K. \& Louail, L. Ab initio investigations of structural, elastic and electronic properties of $\mathrm{ZnSiP}_{2}$ : Pressure effect. Comp. Mater. Sci. 65, 520-527 (2012).

24. Spring-Thorpe, A. J. \& Pamplin, B. R. Growth of some single crystal II-IV-V semiconducting compounds. J. Cryst. Growth 3-4, 313-316 (1968).

25. Prescher, C. \& Prakapenka, V. B. DIOPTAS: a program for reduction of twodimensional X-ray diffraction data and data exploration. High. Press. Res. $\mathbf{3 5}$ 223-230 (2015).

26. Hunter, B. A. Rietica-A Visual Rietveld Program, International Union of Crystallography Commission on Powder Diffraction Newsletter No. 20, http://www. rietica.org, (1998).

27. Mao, H. K. Xu, J. \& Bell, P. M. Calibration of the ruby pressure gauge to $800 \mathrm{kbar}$ under quasi-hydrostatic conditions. J. Geophys. Res. 91, 4673-4676 (1986).

28. Gontijo, R. N. et al. Temperature dependence of the double-resonance Raman bands in monolayer MoS 2 . J. Raman Spectrosc. 50, 1867-1874 (2019).

29. Dey, P. et al. Optical coherence in atomic-monolayer transition-metal dichalcogenides limited by electron-phonon interactions. Phys. Rev. Lett. 116, 127402 (2016)
30. Birch, F. Finite elastic strain of cubic crystals. Phys. Rev. 71, 809-824 (1947).

31. Peña-Pedraza, H., López-Rivera, S. A., Martin, J. M., Delgado, J. M. \& Power, C. Crystal and phonon structure of $\mathrm{ZnSiP}_{2}$ a III-IV-V $\mathrm{V}_{2}$ semiconducting compound. Mater. Sci. Eng. B 177, 1465-1469 (2012).

32. Bernard, J. E. \& Zunger, A. Ordered-vacancy-compound semiconductors: pseudocubic Cdln $2 \mathrm{Se}_{4}$. Phys. Rev. B 37, 6835-6856 (1988).

33. Zunger, A. Order-disorder transformation in ternary tetrahedral semiconductors. Appl. Phys. Lett. 50, 164-166 (1987).

34. Burlakov, I. I., Raptis, Y., Ursaki, V. V., Anastassakis, E. \& Tiginyanu, I. M. Orderdisorder phase transition in $\mathrm{CdAl}_{2} \mathrm{~S}_{4}$ under hydrostatic pressure. Solid State Commun. 101, 377-381 (1997).

35. Werthamer, N. R., Helfand, E. \& Hohenberg, P. C. Temperature and purity dependence of the superconducting critical field, $\mathrm{Hc}_{2}$. III. electron spin and spin-orbit effects. Phys. Rev. 147, 295-302 (1966).

36. Tafti, $F$. F. et al. Sudden reversal in the pressure dependence of $T_{c}$ in the ironbased superconductor $\mathrm{KFe}_{2} \mathrm{As}_{2}$. Nat. Phys. 9, 349-352 (2013).

37. Tafti, $F$. F. et al. Sudden reversal in the pressure dependence of $T_{c}$ in the ironbased superconductor $\mathrm{CsFe}_{2} \mathrm{As}_{2}$ : a possible link between inelastic scattering and pairing symmetry. Phys. Rev. B 89, 134502 (2014).

38. Pang, G. M. et al. Nodeless superconductivity in noncentrosymmetric $\mathrm{PbTaSe}_{2}$ single crystals. Phys. Rev. B 93, 060506(R) (2016).

39. Yesudhas, N. Y. S. et al. Origin of superconductivity and giant phonon softening in TllnTe2 under pressure. Preprint at https:/arxiv.org/abs/2003.09804 (2020). 